\title{
GMR
}

\section{Identification of a mutation in Hepatitis B virus surface antigen capable of evading ELISA screening}

\author{
Y. Cui, T. Zhang, Y. Yan and K. Liu \\ Department of Blood Transfusion, Zhumadian Central Hospital, Zhumadian, \\ Henan Province, China \\ Corresponding author: Y. Cui \\ E-mail: cuiyunxia2015@163.com
}

Genet. Mol. Res. 15 (3): gmr. 15037582

Received September 3, 2015

Accepted December 8, 2015

Published July 15, 2016

DOI http://dx.doi.org/10.4238/gmr.15037582

Copyright (C) 2016 The Authors. This is an open-access article distributed under the terms of the Creative Commons Attribution ShareAlike (CC BY-SA) 4.0 License.

\begin{abstract}
Hepatitis B virus (HBV) infection can cause HBVrelated cirrhosis, liver failure, and hepatocellular carcinoma. At present, a hepatitis B surface antigen (HBsAg) blood test is the primary clinical and diagnostic marker for the identification of a chronic HBV infection. In the current study, we isolated a novel HBV mutant from a chronic HBV patient, capable of causing a false negative test result for most (7 of 8) commercial HBsAg ELISA kits. DNA sequencing of the HBsAg region of this HBV mutant revealed two novel mutation sites that resulted in a Thr-to-Met substitution at amino acid (aa) position 118 and a Lys to Asn substitution at aa position 122 of HBsAg. Moreover, a mutagenesis assay showed that the aa118 (Thr to Met) mutation was the leading cause of the false negative results from the HBsAg ELISA tests. The false negative result was restored, in that the mutation was correctly detected, when the Thr at aa position 118 of this
\end{abstract}

Genetics and Molecular Research 15 (3): gmr.15037582 
mutated HBsAg was reconstituted. In conclusion, our study revealed a novel aa118 Met mutation of HBsAg HBV that will benefit the future development of HBV diagnosis.

Key words: Hepatitis B virus; HBsAg; ELISA; HBV mutation; Blood screening

\section{INTRODUCTION}

The hepatitis B virus (HBV) is a worldwide public health problem, and the number of chronic infected cases of HBV is estimated to be between 300 million and 350 million globally (Lee 1997; Krajden et al., 2005). Chronic HBV infection is responsible for at least one million deaths annually (Dienstag 2008), and without medical intervention, 15 to $40 \%$ of chronic HBV cases will develop into HBV-related cirrhosis, liver failure, or end-stage liver disease, requiring liver transplantation, as well as hepatocellular carcinoma (Krajden et al., 2005).

Based on seroprevalence data, China is considered one of the most highly endemic areas for HBV in the world (Ott et al., 2012). Epidemiology studies suggest that HBV infection in China is up to 7\% in adults (Ott et al., 2012). Moreover, because China has the largest population in the world, the demand for blood transfusion during surgery, as well as other blood-related products for medical needs, is extremely high. Therefore, HBV infection caused by blood transfusion or from the use of HBV-contaminated blood products is another major transmission route, in addition to transmission via sexual contact, drug injection and vertical transmission from an infected mother to an unborn baby (Buddeberg et al., 2008; Toy et al., 2008). This is especially true when the blood was collected from an HBV-infected donor during a period when it could not be detected, or when the donor was infected by a mutated HBV strain that can lead to false negative results during routine HBV screening (Candotti and Allain 2009). For this reason, accurate HBV screening is necessary to ensure safe blood transfusion and good public health.

Currently, enzyme linked immunosorbent assay (ELISA)-based screening for Hepatitis B surface antigen (HBsAg) is the primary method for identifying individuals with chronic HBV infection (Shepard et al., 2006). HBV is a member of the Hepadnaviridae family of viruses, and has a double-stranded DNA genome of approximately $3200 \mathrm{bp}$, containing four partiallyoverlapping open reading frames (ORFs) (Lee 1997; Krajden et al., 2005; Dienstag 2008). These ORFs code for envelope, core (precore/core), polymerase and X proteins (Krajden et al., 2005). During HBV infection, HBsAg (a product of the envelope ORF) is produced in excess amounts and secreted into the blood from virus-infected liver cells (Krajden et al., 2005). Therefore, HBsAg has been used as one of the main clinical markers to indicate acute or chronic infection and prevalence to HBV infection (Shepard et al., 2006).

Many mutations affecting the antigenicity of HBsAg have been reported, and those HBV mutants were found to be associated with either immune escapes or diagnostic failures due to their impaired binding ability for anti-HBV antibodies (Carman et al., 1990; Carman 1997; Tian et al., 2007). Therefore, false negative results for chronic HBV infection due to these HBsAg variations is a major concern for the safety of blood transfusion and the use of other blood products. In this study, we identified a blood sample from a donor that demonstrated a false negative result during HBsAg ELISA screening. Analysis of the HBV DNA sequence

Genetics and Molecular Research 15 (3): gmr.15037582 
of the HBsAg region of this HBV isolate indicated two mutation sites [a threonine (Thr)-tomethionine (Met) substitution at amino acid (aa) position 118 and a lysine (Lys)-to-asparagine (Asn) substitution at aa position 122] that could be responsible for evading ELISA detection. A subsequent mutagenesis study demonstrated that the aa118 Met mutation of HBsAg was the major cause of the false negative ELISA test results. In summary, our data will benefit the future development of improved methods for HBV diagnosis.

\section{MATERIAL AND METHODS}

\section{Ethics statement}

Institutional Ethics Board approval was obtained from the Medical Ethics Committee, Zhumadian Central Hospital. All participating patients were informed of the purpose of this study and written consents were obtained from all participants.

\section{Serum DNA extraction, PCR cloning and plasmids}

DNA was extracted from a serum sample from a chronically infected HBV individual using a QIAamp DNA Mini Kit (Qiagen, Valencia, CA, USA) according to manufacturer protocols. The DNA was used as a template for cloning the HBsAg DNA fragment by PCR, using Phusion high fidelity DNA polymerase (New England Biolabs, Ipswich, MA, USA) according to manufacturer protocols. Primers for PCR were designed according the HBV genotype B and C, and were synthesized by Invitrogen China (Invitrogen China, Shanghai, China). Primer sequences are listed in Table 1. PCR products were purified using a Gel Purification Kit (Takara, Dalian, China) and ligated into the pIRES-Neo3 vector (Clontech Laboratories China, Beijing, China) to generate expression of the secreted form of the recombined HBsAg protein, as previously described (Dai et al., 2008). All plasmids for mammalian expression were transformed to $E$. coli $\mathrm{DH} 5 \alpha$ via heat-shock transformation. $E$. coli DH5 $\alpha$ cells were maintained in-house. All vectors were confirmed via DNA sequencing performed by Genescript (Genescript, Nanjing, China).

\begin{tabular}{|c|c|c|c|}
\hline Primers & Sequence (5' to 3') & Target gene & Construct name \\
\hline $\begin{array}{l}\text { Clone_F } \\
\text { Clone_R }\end{array}$ & $\begin{array}{l}\text { TTAGCTAGCGCCGCCACCATGGAGAACCCATCAGG } \\
\text { CTCGGATCCTTAAATGTATACCCAAAGAC }\end{array}$ & Cloning of $\mathrm{HBsAg}$ & pIRES-Neo3-HBsAg-M \\
\hline $\begin{array}{l}\text { 118(Thr)_F } \\
118(\mathrm{Thr}) \_\mathrm{R}\end{array}$ & $\begin{array}{l}\text { TCATCAACAACCAGC } \underline{\boldsymbol{A} \boldsymbol{A} \boldsymbol{G} \text { GGACCATGCAAATTTGC }} \\
\text { ATGGTCC } \underline{\boldsymbol{C T} T \boldsymbol{T} \text { GCTGGTTGTTATGA }}\end{array}$ & $\begin{array}{l}\text { Introducing T-to-A mutation at nt } 353 \\
\text { (Met-to-Thr substitution at aa 118) }\end{array}$ & pIRES-Neo3-HBsAg-M118Thr \\
\hline $\begin{array}{l}\text { 122(Lys)_F } \\
122 \text { (Lys)_R }\end{array}$ & $\begin{array}{l}\text { ACCGGACCATGC } \overline{\boldsymbol{A A \boldsymbol { A } A} A C C T G C A C A A C T} \\
\text { AGTTGTGCAGGT } \underline{\boldsymbol{T} \boldsymbol{T} T \text { GCATGGTCCGGT }}\end{array}$ & $\begin{array}{l}\text { Introducing C-to-A mutation at nt } 365 \\
\text { (Asn-to-Lys substitution at aa 122) }\end{array}$ & pIRES-Neo3-HBsAg-M122Lys \\
\hline
\end{tabular}

\section{Cells and chemicals}

HEK293T cells, maintained in Dulbecco's Modified Eagle Medium (DMEM) medium supplemented with 10\% fetal bovine serum (Life Technologies, Carlsbad, CA, USA), were used for plasmid transfection and protein expression. Lipofectamine ${ }^{\mathrm{TM}} 2000$ (Life Technologies) was used for transfection according to the manufacturer protocol. Forty-eight hours after the transfection, the supernatant containing the recombinant protein expressed from the plasmids was collected and used in the ELISAs. 


\section{Western blot analysis}

SDS-PAGE and western blotting were performed as previously described (Patel et al., 2010; Nan et al., 2012). Briefly, HEK293T cells that were transfected with different plasmids were lysed in Laemmli sample buffer and subjected to SDS-PAGE. The separated proteins were then transferred onto a PVDF membrane and probed with mouse monoclonal antibodies against HBsAg (Jingtiancheng Biotech, Beijing China) and tubulin (Sigma, St. Louis, MO, USA), followed by a goat anti-mouse IgG secondary antibody conjugated to horseradish peroxidase (Sigma). The signal was detected using a chemiluminescence substrate and was digitally recorded using a ChemiDoc XRS imaging system (Bio-Rad Laboratories, Hercules, CA, USA).

\section{HBsAg diagnosis ELISA kits and standard samples (Quality Control)}

Eight commercial HBsAg diagnosis ELISA kits from different manufacturers were included in this study. However, the full names of these companies will not be disclosed here due to conflicts of interest between the manufacturers. HBsAg standard samples (Quality Control) were obtained from the Clinical Testing Center, China Ministry of Health. All ELISA tests were conducted according to manufacturer instructions for each kit and were repeated at least three times.

\section{Statistical analysis}

Data are reported as means \pm SE. Differences in indicators between samples was assessed using the Student $t$-test. A two-tailed $\mathrm{P}$ value of less than 0.05 was considered significant.

\section{RESULTS}

\section{Identification of a novel HBV isolate that evaded detection by most ELISA kits}

During routine HBV screening of blood samples from donors in our department, we found one blood sample that demonstrated inconsistent results (one positive and one negative) from two HBsAg ELISA kits from two different manufacturers. Previous reports have described HBV mutants that were able to escape detection using existing $\mathrm{HBsAg}$ ELISA kits (Weber 2006; Echevarria and Avellón 2008), leading to safety concerns. We therefore conducted a series of tests using several different ELISA kits, as well as a PCRbased HBV DNA detection method, to determine whether the blood sample was actually positive for HBV. Our test results showed that this blood sample was indeed positive for HBsAg, but that this HBV isolate was able to evade detection using most of the commercial ELISA kits for HBsAg (Figure 1). Of the eight ELISA kits used in this study, only one (No. 6) was able to detect this HBV mutant (Figure 1). Moreover, even for the kit that was able to detect the mutated HBsAg, the $\mathrm{S} / \mathrm{N}$ ratio was much lower than for the HBsAg QC sample (Figure 1), suggesting the antibody used to coat the ELISA plate was only able to partially recognize the mutated HBsAg.

Genetics and Molecular Research 15 (3): gmr.15037582 


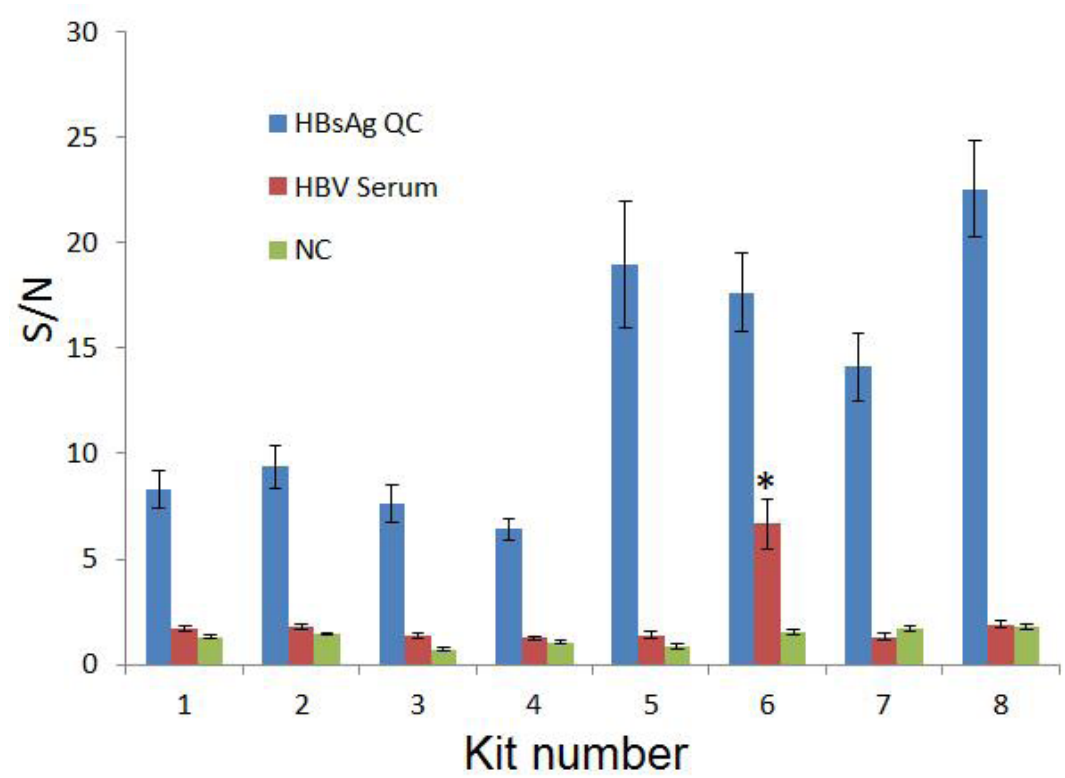

Figure 1. Detection of a novel HBsAg mutant that evaded detection by HBsAg ELISA. Eight commercial ELISA kits for HBsAg from different manufacturers (assigned No. 1 to No. 8) were used to test serum samples for the novel HBsAg mutant. The Quality Control sample $(0.5 \mathrm{mg})$ and negative control from each ELISA kit were included as positive and negative controls, respectively. $\left({ }^{*} \mathrm{P}<0.05\right)$.

\section{Expression of the mutated HBsAg from plasmids demonstrated similar ELISA testing results to the original serum sample}

To identify the sequence location of the mutation sites that caused the false negative HBsAg ELISA test result, the whole HBsAg region of this novel HBV isolate was cloned into a mammalian expression vector, pIRSE-Neo3, and transfected into HEK293T cells to express the recombinant protein, $\mathrm{HBsAg}$ (rHBsAg). Western blotting using an anti-HBsAg antibody confirmed successful expression of the recombinant protein (Figure 2A). The same set of eight commercial HBsAg ELISA kits that were used for the initial tests were then used to determine whether rHBsAg was able to mimic the HBsAg isolated from the original blood sample. Our data demonstrated similar ELISA results for both the serum HBsAg as well as the recombinant HBsAg expressed from HEK293T cells (Figure 2B), indicating that the mutation was successfully introduced into the plasmid and could be used in a mutagenesis assay.

\section{A 118 Thr-to-Met mutation of HBsAg was the cause of the false negative ELISA test result}

After confirming that plasmids expressing rHBsAg could be used in ELISA tests, DNA sequencing was conducted to identify the mutation site that led to the false negative data. Two unique mutation sites [at nucleotide (nt) positions 353 and 365] of HBsAg were identified. An A-to-T mutation at nt 353 led to a Thr-to-Met substitution at amino acid (aa) position 118, while an A-to-C mutation at nt365 led to an Asn-to-Lys substitution at aa position 
122. Further testing was required to determine which of these two mutation sites was the cause of the false negative result is. Two primer pairs were designed (Table 1) to introduce point mutations that would revert the proteins back to wild type. Two constructs, pIRES-Neo3HBsAg-M118Thr (containing the Met-to-Thr substitution at aa position 118 of the mutated HBsAg) and pIRES-Neo3-HBsAg-M122Lys (containing the Asn-to-Lys substitution at aa 122 of the mutated HBsAg), were generated and transfected into HEK293T cells. Western blot analysis confirmed successful expression of the plasmids (Figure 3A), and supernatants from the transfected cells were used for ELISA tests, using the same eight kits.

Restoring the Lys at aa position 122 (HBsAg-M122Lys; mutated HBsAg bearing 122 Lys) still demonstrated the same false negative results as seen with the mutated HBsAg (HBsAg-M), suggesting that the 122 Lys mutation did not play significant role in masking the mutation from the HBsAg ELISA diagnosis (Figure 3B). However, when the Met was restored at aa postion 118 (HBsAg-M118Thr; mutated HBsAg bearing 118Thr), all ELISA kits were able to detect the mutation, indicating that the aa118 Met mutation is the determining factor for the false negative results obtained using most of the HBsAg ELISA kits (Figure 3B). Taken together, our data show that the Met at aa position 118 changed the antigenicity of $\mathrm{HBsAg}$, leading to false negative results from most commercial HBsAg ELISA kits.

A

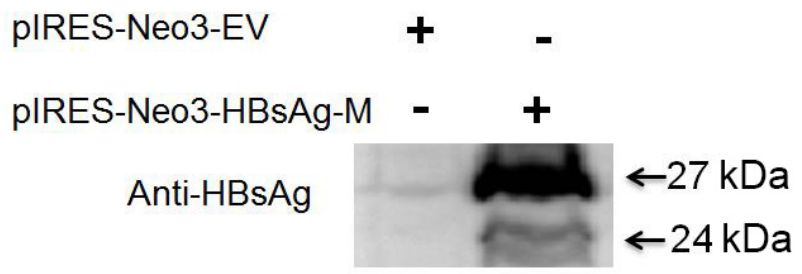

Anti-Tubulin

B

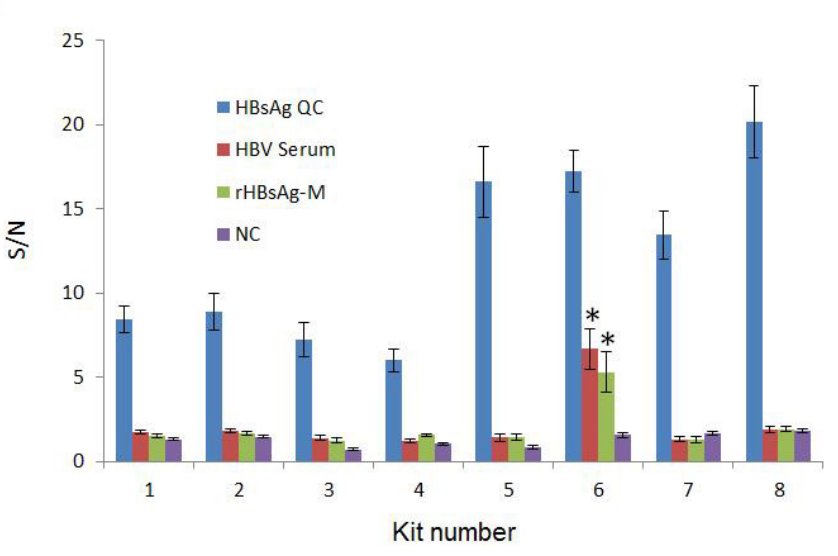

Figure 2. Expression of recombinant HBsAg mutants and ELISA tests for recombinant HBsAg. A. Expression of HBsAg plasmids in HEK293T cells. HEK293T cells were transfected with pIRES-Neo3 vector or pIRES-Neo3 HBsAg-M for $48 \mathrm{~h}$, then lysates were harvested for western blot using an anti-HBsAg antibody. B. ELISA for the HBsAg mutant expressed from plasmids and in blood samples. The Quality Control sample $(0.5 \mathrm{mg})$ and negative control from each ELISA kit were included as positive and negative controls, respectively. $(* \mathrm{P}<0.05)$.

Genetics and Molecular Research 15 (3): gmr.15037582 


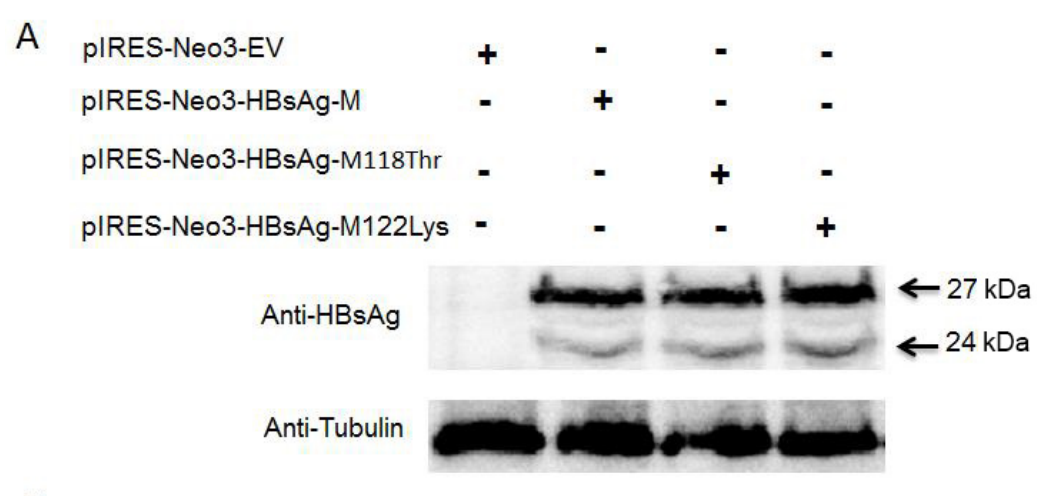

B

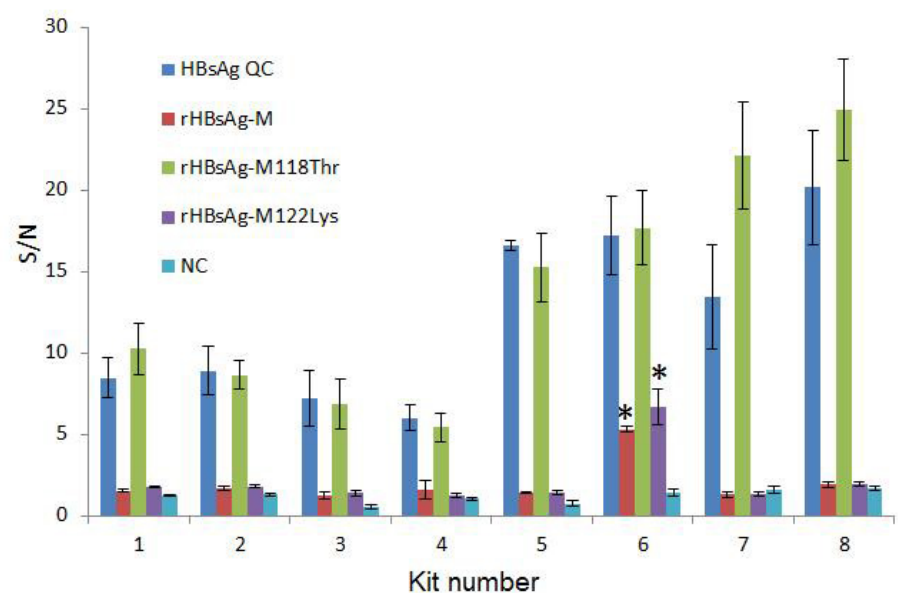

Figure 3. Thr-to-Met mutation at aa position 118 of HBsAg leads to false-negative results in HBsAg ELISA screening. A. Expression of different HBsAg constructs (HBsAg-M, HBsAg-M118Thr and HBsAg-M122Lys) in HEK293T cells. B. ELISA screening for the different recombinant HBsAg proteins. The three HBsAg mutants were tested using the ELISA kits, along with appropriate controls. The Quality Control sample $(0.5 \mathrm{mg})$ and negative control from each ELISA kit were included as positive and negative controls, respectively. ${ }^{*} \mathrm{P}<0.05$.

\section{DISCUSSION}

During HBV replication, three different HBV surface antigens (small, middle and large) are generated that share a common C-terminal (226 aa). The small HBV surface antigen (generally defined as HBsAg) is 226 aa in length and is the major component of the viral envelope (Tian et al., 2007). Further analysis of HBsAg indicates that the major hydrophilic region (MHR) between aa 99 and 169 bears conformational epitopes that represent the major target for neutralizing antibodies (Seddigh-Tonekaboni et al., 2000; Tian et al., 2007).

$\mathrm{HBV}$ is a DNA virus; however, unlike other DNA viruses such as Herpesvirus or vaccinia virus, the replication of HBV comprises a unique stage that involves reverse transcriptase (Radziwill et al., 1990; Bartholomeusz et al., 2004). The HBV-encoded polymerase is a novel, multifunctional reverse transcriptase composed of a reverse transcriptase (RT) domain (Toh et al., 1983), an RNase H domain (Radziwill et al., 1990), and a unique (N)-terminal 
domain called the terminal protein (TP), which functions as a protein primer (Bartenschlager and Schaller 1988). The reverse transcriptase domain of HBV polymerase shows significant homology to retroviral RTs (Radziwill et al., 1990; Bartholomeusz et al., 2004). As a result, HBV reverse transcriptase has a high mutation rate due to the lack of proof-reading activity, which is the main cause of HBV mutations (Deng and Tang 2011). Moreover, the application of antivirus therapy as well as large-scale vaccinations have resulted in selective pressure for HBV to continually mutate, resulting in drug-resistant and immune escape mutations (Zuckerman 2000; Shaw et al., 2006).

In this study, we identified two mutation sites located at amino acid (aa) positions 118 and 122 of HBsAg that could potentially cause the false negative results observed during ELISA screening. A mutagenesis study demonstrated that a Met-to-Thr substitution at aa position 118 was able to restore antigen recognition by all HBsAg ELISA kits used in this study, while an Asnto-Lys substitution at aa position 122 had minimal effect. These data suggest that the major cause of the false negative results is a Thr-to-Met mutation at aa position 118. The major neutralizing targets of HBsAg are conformational epitopes located between aa positions 99 and 169 (SeddighTonekaboni et al., 2000; Tian et al., 2007), making it possible that the Thr-to-Met substitution at aa position 118, which lies within this region, will affect the three-dimensional confirmation of HBsAg, and therefore its antigenicity. Seven of the ELISA kits used in the current study failed to detect the HBsAg 118 Met mutant, but one kit was able to recognize it, suggesting that a different antibody may have been used to coat the ELISA plate.

The small S gene (HBsAg) of HBV is fully embedded in the polymerase gene. HBV mutations in the $\mathrm{S}$ gene have not only resulted in false negative results from some diagnostic kits due but may also be linked to drug resistance (Mizuochi et al., 2006; Deng and Tang 2011). Our data suggest that the aa118 Met mutation in HBsAg also resulted in a Gln to His substitution at aa position 471 of the HBV polymerase. However, due to the limited blood sample we obtained from the original donor, we were unable to conduct further analysis, and whether this HBV polymerase mutation affects the replication of HBV is still unknown.

Manufacturers of HBsAg ELISA kits have improved the antibodies used to coat the plates in an attempt to prevent false negative results. However, HBV is a virus that is capable of mutating frequently due to the utilization of reverse transcriptase, and could therefore generate new mutations to evade detection both from a host immune system and from an in vitro diagnosis product. Therefore, frequent monitoring of new HBV mutations and evaluation of their effects on current diagnosis tools are important for public health. In summary, data from the current study will provide valuable information to enable further improvement of current HBsAg diagnosis methods.

\section{Conflicts of interest}

The authors declare no conflict of interest.

\section{REFERENCES}

Bartenschlager R and Schaller H (1988). The amino-terminal domain of the hepadnaviral P-gene encodes the terminal protein (genome-linked protein) believed to prime reverse transcription. EMBO J. 7: 4185-4192.

Bartholomeusz A, Tehan BG and Chalmers DK (2004). Comparisons of the HBV and HIV polymerase, and antiviral resistance mutations. Antivir. Ther. (Lond.) 9: 149-160.

Genetics and Molecular Research 15 (3): gmr.15037582 
Buddeberg F, Schimmer BB and Spahn DR (2008). Transfusion-transmissible infections and transfusion-related immunomodulation. Best Pract. Res. Clin. Anaesthesiol. 22: 503-517. http://dx.doi.org/10.1016/j.bpa.2008.05.003

Candotti D and Allain JP (2009). Transfusion-transmitted hepatitis B virus infection. J. Hepatol. 51: 798-809. http:// dx.doi.org/10.1016/j.jhep.2009.05.020

Carman WF (1997). The clinical significance of surface antigen variants of hepatitis B virus. J. Viral Hepat. 4 (Suppl 1): 11-20. http://dx.doi.org/10.1111/j.1365-2893.1997.tb00155.x

Carman WF, Zanetti AR, Karayiannis P, Waters J, et al. (1990). Vaccine-induced escape mutant of hepatitis B virus. Lancet 336: 325-329. http://dx.doi.org/10.1016/0140-6736(90)91874-A

Dai JW, Liu SC, Hao LL, Zhang YL, et al. (2008). Simultaneous expression of growth hormone releasing hormone (GHRH) and hepatitis B surface antigen/somatostatin (HBsAg/SS) fusion genes in a construct in the skeletal muscle enhances rabbit weight gain. Anim. Biotechnol. 19: 104-116. http://dx.doi.org/10.1080/10495390801888328

Deng L and Tang H (2011). Hepatitis B virus drug resistance to current nucleos(t)ide analogs: Mechanisms and mutation sites. Hepatol. Res. 41: 1017-1024. http://dx.doi.org/10.1111/j.1872-034X.2011.00873.x

Dienstag JL (2008). Hepatitis B virus infection. N. Engl. J. Med. 359: 1486-1500. http://dx.doi.org/10.1056/ NEJMra0801644

Echevarría JM and Avellón A (2008). Improved detection of natural hepatitis B virus surface antigen (HBsAg) mutants by a new version of the VITROS HBsAg assay. J. Med. Virol. 80: 598-602. http://dx.doi.org/10.1002/jmv.21146

Krajden M, McNabb G and Petric M (2005). The laboratory diagnosis of hepatitis B virus. Can. J. Infect. Dis. Med. Microbiol. 16: 65-72.

Lee WM (1997). Hepatitis B virus infection. N. Engl. J. Med. 337: 1733-1745. http://dx.doi.org/10.1056/ NEJM199712113372406

Mizuochi T, Okada Y, Umemori K, Mizusawa S, et al. (2006). Evaluation of 10 commercial diagnostic kits for in vitro expressed hepatitis B virus (HBV) surface antigens encoded by HBV of genotypes A to H. J. Virol. Methods 136: 254-256. http://dx.doi.org/10.1016/j.jviromet.2006.03.022

Ott JJ, Stevens GA, Groeger J and Wiersma ST (2012). Global epidemiology of hepatitis B virus infection: new estimates of age-specific HBsAg seroprevalence and endemicity. Vaccine 30: 2212-2219. http://dx.doi.org/10.1016/j. vaccine. 2011.12 .116

Nan Y, Wang R, Shen M, Faaberg KS, et al. (2012). Induction of type I interferons by a novel porcine reproductive and respiratory syndrome virus isolate. Virology 432: 261-270. http://dx.doi.org/10.1016/j.virol.2012.05.015

Patel D, Nan Y, Shen M, Ritthipichai K, et al. (2010). Porcine reproductive and respiratory syndrome virus inhibits type I interferon signaling by blocking STAT1/STAT2 nuclear translocation. J. Virol. 84: 11045-11055. http://dx.doi. org/10.1128/JVI.00655-10

Radziwill G, Tucker W and Schaller H (1990). Mutational analysis of the hepatitis B virus P gene product: domain structure and RNase H activity. J. Virol. 64: 613-620.

Seddigh-Tonekaboni S, Waters JA, Jeffers S, Gehrke R, et al. (2000). Effect of variation in the common "a" determinant on the antigenicity of hepatitis B surface antigen. J. Med. Virol. 60: 113-121. http://dx.doi.org/10.1002/(SICI)10969071(200002)60:2<113::AID-JMV2>3.0.CO;2-0

Shaw T, Bartholomeusz A and Locarnini S (2006). HBV drug resistance: mechanisms, detection and interpretation. $J$. Hepatol. 44: 593-606. http://dx.doi.org/10.1016/j.jhep.2006.01.001

Shepard CW, Simard EP, Finelli L, Fiore AE, et al. (2006). Hepatitis B virus infection: epidemiology and vaccination. Epidemiol. Rev. 28: 112-125. http://dx.doi.org/10.1093/epirev/mxj009

Tian Y, Xu Y, Zhang Z, Meng Z, et al. (2007). The amino Acid residues at positions 120 to 123 are crucial for the antigenicity of hepatitis B surface antigen. J. Clin. Microbiol. 45: 2971-2978. http://dx.doi.org/10.1128/JCM.00508-07

Toh H, Hayashida H and Miyata T (1983). Sequence homology between retroviral reverse transcriptase and putative polymerases of hepatitis B virus and cauliflower mosaic virus. Nature 305: 827-829. http://dx.doi. org/10.1038/305827a0

Toy M, Veldhuijzen IK, Mostert MC, de Man RA, et al. (2008). Transmission routes of hepatitis B virus infection in chronic hepatitis B patients in The Netherlands. J. Med. Virol. 80: 399-404. http://dx.doi.org/10.1002/jmv.21098

Weber B (2006). Diagnostic impact of the genetic variability of the hepatitis B virus surface antigen gene. J. Med. Virol. 78 (Suppl 1): S59-S65. http://dx.doi.org/10.1002/jmv.20610

Zuckerman AJ (2000). Effect of hepatitis B virus mutants on efficacy of vaccination. Lancet 355: 1382-1384. http://dx.doi. org/10.1016/S0140-6736(00)02132-2

Genetics and Molecular Research 15 (3): gmr.15037582 